\title{
IMPLEMENTING AN ONLINE TRAINING PROGRAMME IN PRECISION MEDICINE FOR PRIMARY CARE PROFESSIONALS: A MULTI-METHOD APPROACH
}

\author{
Sharon Mitchell ${ }^{1}$, Evrim Jaccard ${ }^{2}$, Regula Cardinaux ${ }^{2}$, Prune Collombet ${ }^{3}$, Jacques Cornuz ${ }^{4}$, \\ Gérard Waeber ${ }^{2}$, Idris Guessous ${ }^{3}$ and Sissel Guttormsen ${ }^{1}$ \\ ${ }^{l}$ Institute for Medical Education, Medical Faculty, University of Bern, Mittelstrasse 43, 3012 Bern, Switzerland (CH) \\ ${ }^{2}$ Department of medicine, University Hospital CHUV-Lausanne, $\mathrm{CH}$, \\ ${ }^{3}$ Division and department of primary care medicine, Geneva University Hospitals and Faculty of Medicine, Geneva, CH \\ ${ }^{4}$ Unisanté, Faculty of biology and medicine, University of Lausanne, Rue du Bugnon 44, 1011 Lausanne, CH
}

\begin{abstract}
Never before have expectations of healthcare professionals been more challenged by complex diseases and more educated patients in increasingly consumer driven healthcare systems. A new era of Precision Medicine (PM) is gaining momentum to bring tailored interventions to patients, informed by genomics, lifestyle and environment. The delivery of a more precision approach requires targeted training to frontline health care professionals. The objective of this paper is to report on the structured design and planning of online training modules for Primary Care Professionals (PCPs) specifically targeted at family doctors, pharmacists and advanced practice nurses. We report on initial findings of an ongoing mixed-method approach initiating applied research in a process where each method informs, guides and refines the design of the next step. Kern's 6 Steps method serves as development framework. Literature and experts' reviews supported clarification of learning objectives, focus groups, guided by Kirkpatrick's levels of evaluation supports refinements of content and form. The findings to date support the need to use methods that will challenge pre-existing assumptions, employ best practice approaches informed by evidence and ensure the development of a quality online training programme acceptable and applicable to the defined target groups.
\end{abstract}

\section{KEYWORDS}

Online Training, Precision Medicine, Primary Care Professionals, Needs Assessment, Blended Learning

\section{INTRODUCTION}

This approach for needs assessment, our first data on acceptance from PM experts and PCPs, and planned next steps. The target group identified within 'PCPs' include family doctors, pharmacists and advanced practice nurses, but also other groups of health professionals will be considered.

Precision Medicine (PM) as an era of 'precision' or 'personalisation' in patient care continues to gain momentum within the literature. While traditionally emerged in the study of genomics, PM is rapidly incorporating new domains including precision public health, environment and lifestyle, use of big data and health records (National Library of Medicine 2018) challenging previously conceived definitions of PM as the practice of genomics. Yet infiltration in the use and practice of bringing the right strategy to the right patient, particularly for frontline professionals is needed (Feero 2017). Adapting to changing contexts and new methods to deliver patient care in already challenging healthcare environments is not an easy feat. This project responds to a call from medical experts in Precision Medicine in Switzerland, to design a national training programme that will equip PCPs with the competencies to deliver a precision approach to their patients. It is intended that this training programme will be a blended learning progamme (online and on-site learning) to allow flexible learning environments for busy healthcare professionals.

Online training offers an approach well-fitting with the rapid developments in PM as well as the need for timely updates and the transfer of new knowledge (Ruiz et al 2006). This expanding digital learning era does come with caution and a number of studies have reported the lack of guiding effective instructional design theories in the development of digital learning for health professions education (Dunleavy et al. 2019). The 
importance of applying a conceptual framework for project development, documented within the literature, is therefore a worthy consideration (Bordage 2009).

As an initial step in designing and implementing an online training platform, the project team introduces the use of existing educational frameworks that will guide development. To effectively deliver learning objectives, flexible instructional methods will support learner competency and a blended learning approach (Liu et al, 2016). The design and planning of this project will consider the need for a mix of online and on-site training to support the transfer of knowledge to applied practice (Dunleavy et al 2019). Table 1 shows the adapted model of Kern's 6 step approach to curriculum design and planned steps for project development (Thomas et al, 2016). It underlines the importance of using a general and targeted needs assessment. The paper reports on the first steps of a needs assessment for the target PCP population as well as the first four steps of Table 1.

Table 1. Application of Kern's Six Step Approach to design of a training programme for PCPs

\begin{tabular}{|c|c|}
\hline Kerns 6 Step Approach & Planned Study Phases \\
\hline 1. Problem Identification and General Needs Assessment & Literature Review and Expert Opinion \\
\hline 2. $\quad$ Targeted Needs Assessment & Focus Group Study, Survey to experts \\
\hline 3. Goals and Objectives & $\begin{array}{l}\text { Defining learning objectives with experts } \\
\text { Content development with experts } \\
\text { Content validation in peer review }\end{array}$ \\
\hline 4. Educational Strategies & Literature Review, Expert validation \\
\hline 5. Implementation & $\begin{array}{l}\text { Delivering an online learning platform } \\
\text { Supporting networking } \\
\text { Developing on-site training }\end{array}$ \\
\hline 6. $\quad$ Evaluation and feedback & Participant Evaluation and Field Study \\
\hline
\end{tabular}

The skills required to deliver this project were recognised as an essential first step in building a diverse research team. These skills include experts in the following fields: Precision Medicine, family medicine, pharmacy, nursing, web-design, communication, e-learning and medical education. Regular project meetings have served as an essential point-of-contact to discuss key findings offering diverse perspectives not only from researchers but also through the lens of topic experts and target study participants (Creswell 2009). The research questions for this project are: Q1: What would motivate PCPs to attend online training for PM? Q2: What must be offered in online training programme, to enable PCPs to deliver PM to their patients? Q3: How can we ensure a best practice approach to design an online training programme?

\section{METHODS}

The systematic process of assessing needs is a means to determine the 'gap' between current practices and future expectations, as well as assessing what might be acceptable to a specific target group (Lockyer et al 1998). Figure 1 illustrates a mixed-method approach to needs assessment. Previously published articles advocating the use of multiple methods within Needs Assessment design and delivery (Fraenkel, Wallen \& Hyun 2015) guide this approach. An essential strength is that information is gathered, validated, and refined from different sources offering new perspectives at each stage in the process (Creswell 2009; Keister \& Grames 2012), striking a delicate balance between limitations of qualitative and quantitative research.

As part of the review of the literature, a member of the research team searched through databases including PubMed, Medline, Google (also -Scholar and -books) with the following key terms: Precision Medicine in Primary Care/ Personalised Medicine in Primary Care/ Genomics in Primary Care/ Pharmacogenomics/ Shared decision-making in PM. The researcher gathered and logged publications based on (1) Opinion of relevance, (2) Formal and informal qualitative and quantitative studies, (3) Literature reviews and (4) Year of publication (2014 to date), to gather information on the 'new' field of PM across different sources and evidence (Druery et al 2013). The process documented a list of publications validated by members of the project team.

Expert validation or expert collaboration in qualitative research supports best practices purported within the literature that 'adds validity' and credibility to qualitative inquiry (Creswell \& Miller 2000). With the view of building a working definition of PM for our project, key findings from the literature were discussed with interprofessional experts in order to validate extracted conceptual definitions. The targeted discussion groups included 2 PM experts, a pharmacist and an internist. 



Figure 1. A multi-method approach to Needs Assessment

A framework for the training programme design for the research team shall ensure consideration of essential areas of development within this planning stage. Building upon the review of the literature and expert validation, the research team began the process of assembling key themes requiring further investigation during the qualitative study. In order to extract possible key terms (i.e. learners' motivation) addressing all relevant aspects of the intended training, a suitable educational framework was needed and sought for in the literature.

Focus groups are qualitative methods that have been widely used for the purpose of education needs assessments (Tipping 1998). For the purpose of refining scope within this study, we report on the design and pilot of a question route including main questions and sub-questions, to deliver 2 future inter-professional focus groups. The preparation for the focus groups has followed a design structure as shown in Figure 2 and following best practice approaches in the literature (Stalmeijer 2014).

\begin{tabular}{|c|c|c|c|}
\hline STUDY PURPOSE & TARGET AUDIENCE & METHODOLOGY & FACILITATION \\
\hline $\begin{array}{l}\text { - Explore the topic } \\
\text { - Collect data on research question } \\
\text { - Consider key themes }\end{array}$ & $\begin{array}{l}\text { - Define study population } \\
\text { - Purposeful sampling }\end{array}$ & $\begin{array}{l}\text { - Develop initial question route. } \\
\text { - Pilot questions } \\
\text { - Onsite task planning including } \\
\text { supporting materials }\end{array}$ & $\begin{array}{l}\text { - Assign the focus group team members } \\
\text { (moderator, expert, note-taker) } \\
\text { - Plan a practice session } \\
\text { - Refine documents }\end{array}$ \\
\hline
\end{tabular}

Figure 2. Focus group design adapted from Nagle \& Williams 2013

The question route was developed aligned to the key themes from an educational framework guiding development. 4 members of the research team interviewed target group participants including 2 family doctors, a pharmacist and an applied practice nurse. This method follows a best practice approach to assess how questions are understood as part of the process to refine and improve research design (Adams \& Cox 2008).

A follow up survey to a wider target audience will aim at: validate gathered perspectives of individual experts, test whether gathered perceptions of each target group profession is representative of that profession as a whole and validate to which degree our assumptions for methods of delivery reflect perceived needs. Relevance of content and levels of motivation represent the target audience in general.

\section{RESULTS}

The results of each step from Figure 1 show that within a short time frame an interprofessional project team agreed on clearly aligned stages, each bringing small but relevant insights for the project as a whole.

Table 2. Collation of key terms with perceived importance in Precision Medicine \& expert validation result

\begin{tabular}{ll}
\hline $\begin{array}{l}\text { Key terms linked to Precision Medicine } \\
\text { Result of Literature Scope }\end{array}$ & $\begin{array}{l}\text { Rejection or support } \\
\text { Result of Expert Validation }\end{array}$ \\
\hline Genomics & Yes, should be included \\
\hline Clinical phenotypes & Not needed \\
\hline Biological sampling & Not needed \\
\hline Variability in genes, lifestyle and environment & Yes, should be included \\
\hline Health records and big data & Yes, should be included \\
\hline Customized prevention and treatment strategies & Yes, should be included \\
\hline
\end{tabular}

The review of the literature led to important findings in the scope and breath of the topic of PM as a newly evolving field, and the resulting learning objectives. In establishing the support of an online training programme in PM, the dominant perspective from the medical experts was to focus on the development of 
genomics, a topic synonymous with PM. A review of the literature on PM found additional key terms not isolated to genomics presented in Table 2. In the second step the key terms were validated with PM experts and consensus was reached on terms to include or dismiss as part of a definition of PM for project implementation in a long-term perspective (Table 2).

Selecting a framework for the design of an online training programme was an effort saluted to the wellknown phrase begin with the end in mind, also recognised in curriculum design (Morrison 2003). We found that Kirkpatrick's 4 levels of evaluation, adapted from Barr et al 2006 offered a structured guide to determine overall themes and more specific objectives, see Figure 3.

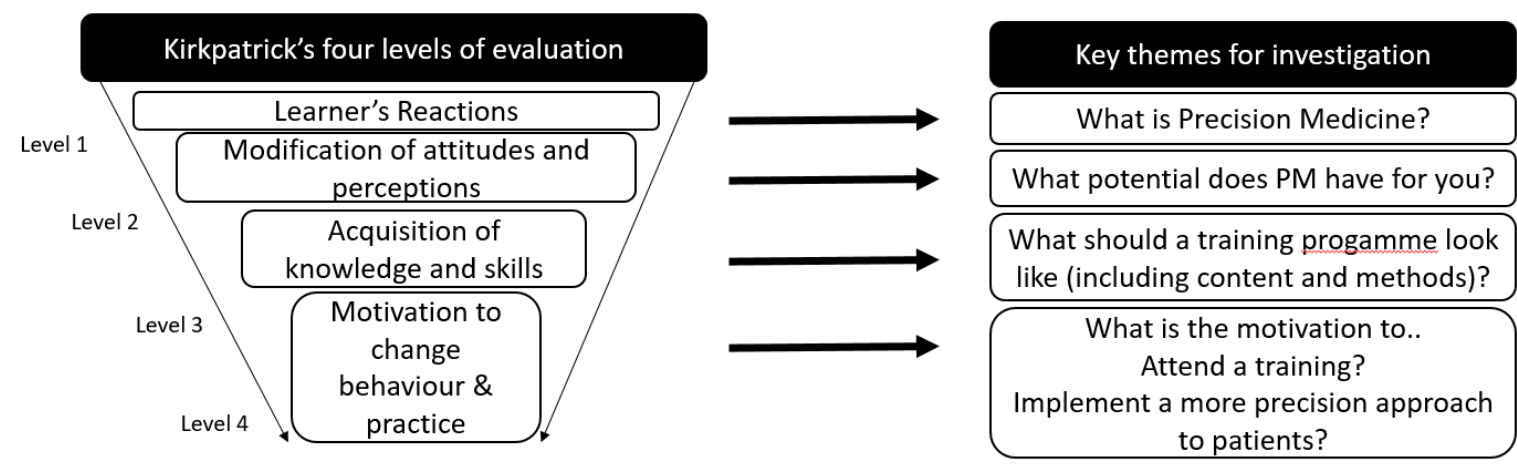

Figure 3. Developing essential objectives to explore 'key themes' derived from Kirkpatrick's levels of evaluation

The focus group design and planning phase followed the insights documented in Figures 2 and 3. Building upon the key themes from Kirkpatrick's model, the research team developed a specific question route covering the four theme questions from the model in Figure 3. Besides validating aspects related to the content, the pilot study showed the importance of assessing the motivation to use an online training for PCPs. PCPs typically spend most of their time in clinics or their doctor's office and the topic of PM is also competing with many new areas and fields of study. Therefore, it does not help to build a quality online training programme, if the target group have no time or motivation to use it. The pilot offered valuable feedback that PCPs are not well informed about PM as this is a novel topic. Hence, the intention to optimise delivery and training of this new knowledge and possible skills to frontline professionals, using the opportunities of online training is assured at this stage of the development. Next steps of the Needs Assessment phase will deliver two focus group studies and a survey. Data of the focus group study will be ready for the conference in November.

\section{DISCUSSION}

This paper reports on the first steps in the design and implementation of an online training programme for PM. We have documented a process, confirming the need for further validation of the research questions through a systematic process for needs assessment (Figure 1). This process offers preliminary answers to the overall research questions.

Q1 addresses motivation to attend an online training programme in PM. Although results are preliminary, we can deduce that PM is a new field understood very differently across healthcare professionals. While participants responded that this may not be a topic relevant for today, the overall trend of responses from our initial pilot support the need for this training. The aspect of motivation and willingness to attend must continue to be monitored, also in terms of the means of knowledge delivery (on-line, mobile or more traditional) that can lead to implications in daily practice. Online delivery is only one part of the answer. $Q 2$ explicitly focuses on content, the driver for success, and the particular granularity and the orientation of the needs of the different professions. The offered content must fit with the needs of the target groups, the clarification of which is the foundation of any curriculum development. Further, the content for development and means of delivery are intrinsically linked. It would be wrong to assume that all content is suitable for online delivery only, or that online training alone will meet the expectations of learners. The success of this training programme will be to strike a balance between the means of delivery, the content for training and the ultimate goal of meeting the expected needs of busy healthcare professionals. $Q 3$ has been a constant thread throughout the research to date 
and our experience shows that the use of existing frameworks has been of immense support in developing a complex online training programme, designed for continuing professional development for different target groups across PCPs.

The review of the literature led to important findings in the scope and breath of the topic of PM as a newly evolving field. While traditionally synonymous in the study of genomics, this is a field that is rapidly emerging into other areas including precision public health, environment and lifestyle, ethics, use of big data and health records, areas that are particularly fitting to frontline healthcare professionals. In establishing the support of an online training programme in PM, the dominant perspective from collaborating medical experts was to focus on the development of genomics. The most notable result has ensured that not only does the agreed definition (and future content of the planned training programme) include genomics but will also address other topics important for the future of PM and the role that PCPs will have in this approach to patient care. While the literature review was of a scoping nature, the expert validation of the finding supports the relevance.

The inclusion of theoretical frameworks, including Kern's Model for curriculum development and Kirkpatrick's 4 levels of evaluation have helped the research team to prescribe specific milestones, align research themes, and support decision making in the project team. Kirkpatrick's model emphasises the importance of the learner at each level taking into account how the learner has reacted, acquired knowledge and skills, and changed behaviour to make a markable difference in the delivery practice performance. The project team will continue to keep the interests of the end users placed at the very centre of the development. Furthermore, the design and planning of 2 focus groups, addressing the French and the German speaking Swiss target groups, has followed a documented process for development, for which the literature provides ample support and evidence. For the purpose of scope within this paper, we are assured of the importance of the essential role of piloting of questions prior to delivery of the focus group. The preliminary results led to extensive discussion, reorganisation and modification of central issues based on the collated responses during this pilot.

The immediate limitations of this paper is that it presents initial findings. Still, we regard the careful consideration of each step in the process to be important and in the end economical, supporting sustainable outcomes. It may be argued that the project is driven from frontline experts of PM with personal interests in dissemination. Yet, the interdisciplinary and multi-professional composition of the project team, as well as the documented development process serve the purpose of avoiding bias. This paper focuses on the online part of the learning programme. Also, the development of the full blended learning programme, integrating online and on-site training shall be given attention as the project develops. Some of the content needs hands on training (e.g. communicating with patients) and exchange of experience between PCPs (e.g. about patients reported needs), which best can be provided with on-site meetings. Further research shall help us find a balance between online and on-site training. It also may be argued that a theoretical framework for developing an online training programme is not an isolated method, however, further findings within this needs assessment fully support this process as a means to provide an intellectual structure that guides the development of the project as a whole.

\section{CONCLUSION}

This paper introduces the first steps taken in the design, planning and implementation of an online learning programme in PM for frontline healthcare professionals. We have reported on our initial findings that support the need to continue our investigations on (1) motivation of our target group (2) relevant content and means of delivery and (3) evidence-based frameworks to ensure acceptability of a high-quality training programme across the target population.

Our initial findings support that both the attractiveness and relevance of the content but also motivation of our target audience to attend a training programme are key elements to successful implementation. Further research aims at documenting these findings as well as implementation steps. The overall success of this programme will be measured by (1) the response and uptake of modules among Swiss frontline professionals, (2) the level of feedback received to disclose relevant information for improvements and (3) monitoring and evaluation of how this training infiltrates into a more precision approach to healthcare delivery. Additional modes of evaluation including the assessment of perceived relevance to daily practice and through subsequent and frequent participation to online modules will offer tangible insight into the 'success' of this training. 
The emphasis on research within the domains of instructional effectiveness and satisfaction of online learning is well reported within the literature. The processes described within this paper respond to the call from systematic findings that more quality data is needed on the implementation and use of education frameworks in designing learning needs assessment, curriculum development and implementation for the purpose of online training. We have documented a multistep approach and use of an evidence-based framework for development to take decisions for implementation. Our first experiences have assured us that this approach is worth the effort. The development of an online interprofessional training programme for (inter-) national use is an expensive mission. The degree of success cannot be a surprise following the final dissemination. We must take every effort to ensure that the steps needed to build a quality, acceptable online training programme worthy of our target audience be built into the process from its inception.

\section{ACNOWLEDGEMENT}

The project team would like to thank Health2030 for their financial support.

\section{REFERENCES}

Adams A \& Cox AL. 2008. Questionnaires, in-depth interviews and focus groups. In: Cairns, P \& Cox, AL. eds. Research Methods for Human Computer Interaction. Cambridge, UK: Cambridge University Press, pp. 17-34

Barr H, Freeth D, Hammick M, Koppel I, Reeves S. 2006. The evidence base and recommendations for interprofessional education in health and social care. Journal of Interprofessional Care. Jan 1;20 (1):75-8.

Bordage G. Conceptual frameworks to illuminate and magnify. Med Educ. 2009;43 (4):312-319. doi:10.1111/j.13652923.2009.03295.x

Creswell JW. 2009. Research Design Qualitative, Quantitative, and Mixed Methods Approaches. Thousand Oaks, CA. SAGE Publications Ltd.

Creswell JW \& Miller DL. 2000. Determining validity in qualitative inquiry. Theory into practice. Aug 1;39 (3):124-30.

Druery J, McCormack N, Murphy S. 2013. Are best practices really best? A review of the best practices literature in library and information studies. Evidence Based Library and Information Practice. Dec 8;8 (4):110-28.

Dunleavy G, Nikolaou CK, Nifakos S, Atun R, Law GC, Car LT. Mobile digital education for health professions: systematic review and meta-analysis by the Digital Health Education Collaboration. Journal of medical Internet research. 2019;21(2).

Feero WG. 2017. Is" Precision Medicine" Ready to Use in Primary Care Practice? Yes: It Offers Patients More Individualized Ways of Managing Their Health. American family physician. Dec 15;96 (12):767-8.

Fraenkel JR., Wallen, NE, \& Hyun HH. 2015. How to Design and Evaluate Research in Education. McGraw-Hill International Edition ed. Vol. Ninth Edition. New York: Mc Graw Hill.

Keister D, Grames H. 2012. Multi-method needs assessment optimises learning. The clinical teacher. Oct; 9(5):295-8.

Liu Q, Peng W, Zhang, F., Hu R, Li Y. and Yan W., 2016. The effectiveness of blended learning in health professions: systematic review and meta-analysis. Journal of medical Internet research, 18(1), p.e2.

Lockyer J, Hanley D, Fidler H, Toews J, Lysholm-Andrews E. 1998. Physician educational needs in osteoporosis: An approach to needs assessment. Journal of Continuing Education in the Health Professions. Jun; 18(3):185-9.

Morrison J. 2003. Ch 4. Evaluation. In Cantillon \& Wood. ABC of Teaching and Learning $2^{\text {nd }}$ Edition. Wiley-Blackwell. Oxford.

National Library of Medicine. 2018. What is precision medicine? Online 24 August 2020 https://ghr.nlm.nih.gov/primer/precisionmedicine/definition

Nagle B. and Williams, N, 2013. Methodology brief: Introduction to focus groups. Center for Assessment, Planning and Accountability, (1-12). Online 24 April 2020 http://www.mmgconnect.com/projects/userfiles/file/focusgroupbrief.pdf

Ruiz JG, Mintzer MJ, Issenberg SB. 2006. Learning objects in medical education. Medical teacher. Jan 1;28 (7):599-605.

Stalmeijer RE, McNaughton N. and Van Mook WN, 2014. Using focus groups in medical education research: AMEE Guide No. 91. Medical teacher, 36(11), pp.923-939.

Thomas PA, Kern DE, Hughes MT, Chen BY. 2016. Curriculum development for medical education. JHU Press: Baltimore, MD, USA.

Tipping, J., 1998. Focus groups: a method of needs assessment. Journal of Continuing Education in the Health Professions, 18(3), pp.150-154 